This is the submitted version of the article:

García Á.M., Corro E.D., Kalbac M., Frank O.. Tuning the electronic properties of monolayer and bilayer transition metal dichalcogenide compounds under direct out-of-plane compression. Physical Chemistry Chemical Physics, (2017). 19. : 13333 - . 10.1039/c7cp00012j.

Available at: https://dx.doi.org/10.1039/c7cp00012j 


\title{
Tuning the electronic properties of monolayer and bilayer transition metal dichalcogenide compounds under direct out-of-plane compression
}

\author{
Ángel Morales-García ${ }^{a *}$, Elena del Corro ${ }^{b *}$, Martin Kalbac ${ }^{b}$, Petr Nachtigall a, Otakar Frank ${ }^{b}$ \\ ${ }^{a}$ Department of Physical and Macromolecular Chemistry, Faculty of Science, Charles University in Prague, Hlavova \\ 2030, 12843 Prague 2, Czech Republic \\ ${ }^{b}$ J. Heyrovsky Institute of Physical Chemistry of the AS CR, v.v.i, Dolejskova 2155/3, 18223 Prague 8, Czech Republic \\ *e-mail: a.moralesg@natur.cuni.cz,edelcorro@quim.ucm.es
}

\begin{abstract}
The band-gap modulation of atomically thin semiconductor transition metal dichalcogenides $\left(M X_{2} ; M=M o\right.$ or $\mathrm{W}, X=\mathrm{S}$ or $\left.\mathrm{Se}\right)$ under direct out-of-plane compression is systematically studied by means of the density functional theory formalism. This stress regime significantly reduces the pressure threshold at which the semimetal state is achieved (2.7-3.1 and 2.0-3.5 GPa for mono- and bilayer systems, respectively). Structural, electronic and bonding properties are investigated for a better understanding of the electronic transitions achieved with compression. A notable relationship with the formal ionic radius $\left(\mathrm{M}^{4+}\right.$ and $\left.\mathrm{X}^{2-}\right)$ is found out. On one hand, the monolayer systems with the smallest transition metal radius $\left(\mathrm{Mo}^{4+}<\mathrm{W}^{4+}\right)$ reach the semimetal state at the lower stress, on the other hand, for bilayer specimens the transition to semimetal is observed earlier for compounds with the smallest chalcogenide radius $\left(\mathrm{S}^{2-}<\mathrm{Se}^{2-}\right)$. Moreover, the appearance of non-covalent interaction $(\mathrm{NCl})$ domains in the semimetal state confirms that the out-of-plane compression promotes the interaction between sulfur atoms in the single layered systems and reduces the interlayer space in bilayer configurations. Our predictions, supported by experimental evidences in the case of monolayered MoS2, open new alternatives for tuning the electronic properties of transition metal dichalcogenides under direct out-ofplane compression.
\end{abstract}




\section{Introduction}

The field of 2D semiconductor transition metal dichalcogenides (TMDs) is being widely investigated due to their outstanding electronic properties, ideal for optoelectronic devices $\left[{ }^{1-2}\right]$. The direct band-gap characteristic of monolayered (1L) TMDs evolves into an indirect one when the sample thickness increases. Tuning the band-gap of TMDs $\left(M X_{2}, M=M o, W\right.$ and $\left.X=\mathrm{S}, \mathrm{Se}\right)$ with the aid of strain constitutes an important strategy to enhance the performance of electronic devices $\left[^{3}\right]$. Recently, several works have reported the strain engineering on monolayered $\mathrm{MoS}_{2}, \mathrm{WS}_{2}, \mathrm{MoSe}_{2}$ and $\mathrm{WSe}_{2}$ systems from an experimental and a theoretical point of view $[4,5,6,7,8,9,10]$ showing the crucial importance that the strain regime and direction may have on the absolute band-gap energy modulation with progressive deformation. Additionally, the strain-tuning gains another degree of freedom in TMD systems with two or more layers through the modification of the interlayer coupling [11,12,13]. The band-gap modulation, therefore, depends mainly on two factors: (i) the size of the system (the strain range required to alter the band-gap is different in monolayer, bilayer (2L) or bulk systems); and (ii) the direction and regime of strain (i.e. uniaxial, biaxial or hydrostatic) $[9,14]$.

Concerning high pressure, several experimental studies coupled with computational predictions have been carried out to investigate multilayered $M X_{2}$ systems under hydrostatic conditions $[11,15,16,17]$. All these high pressure experiments are performed using diamond anvil cell (DAC) devices. A semiconductor to semimetal transition is commonly observed for all compounds at pressure values ranging from 19 to $40 \mathrm{GPa}$ depending on the composition. Nayak et al. [11] reported the study of multilayered $\mathrm{MoS}_{2}$, where a structural lattice distortion (within the same crystal structure) is observed along with an electronic transition from semiconductor to semimetal at $19 \mathrm{GPa}$, with the theoretical prediction of such transition being approximately 24 GPa. The metallization arises when the interlayer space is reduced leading to S-S interactions and to the overlapping of the valence ( $\Gamma$ point in the Brillouin zone) and the conduction (between $\mathrm{K}$ and $\Gamma$ points) bands. The same authors $\left[{ }^{16}\right]$ investigated multilayered $\mathrm{WS}_{2}$, revealing that the band-gap closure is modulated by the increase of the interlayer S-S interactions at pressures above $22 \mathrm{GPa}$ (similar pressure is predicted using first-principles calculations). Other study, published by Zhao et al. $\left[{ }^{15}\right]$, analyzed the behavior of multilayered $\mathrm{MoSe}_{2}$. They also 
found that the pressure induced the metallization, analogously to that observed for $\mathrm{MoS}_{2}$, at 40 $\mathrm{GPa}$, in good agreement with the theoretical predictions (41 GPa). Previously to these studies, Liu et al. $\left.{ }^{[17}\right]$ reported the metallization of multilayered $\mathrm{WSe}_{2}$ at $38 \mathrm{GPa}$, slightly above theoretical predictions (35 GPa), with this electronic change accompanied by an isostructural phase transition. By comparing all these previous studies, it is noted that the sulfide compounds $\left(\mathrm{MS}_{2}\right)$ undergo the transition to a metal state at substantially lower pressures than selenide ones $\left(\mathrm{MSe}_{2}\right)$.

Among all monolayered TMDs compounds, $1 \mathrm{~L}-\mathrm{MoS}_{2}$ has been investigated under different strain regimes. Concerning high pressure studies, we find that the effect of hydrostatic pressure on the TMDs' electronic structure has been analyzed: first the direct band-gap energy increases $\left.{ }^{[14}\right]$ while the indirect one is reduced, leading to a direct to indirect band-gap transition at 23 $\mathrm{GPa}$; then the semiconducting to semimetal transition is theoretically predicted to happen at $\sim 68 \mathrm{GPa}$. More recently, it has been demonstrated that the application of small out-of-plane axial compression can be used for strain engineering of the electronic structure of $1 \mathrm{~L}-\mathrm{MoS}_{2}\left[{ }^{9}\right]$. This experimental study combined with first-principles calculations reports that the transition from direct to indirect band-gap semiconductor takes place at only $0.5 \mathrm{GPa}$, followed by a transition to the semimetal state at values of compression below $3 \mathrm{GPa}$. It must be noted that the stress along the out-of-plane direction required to metallize the $1 \mathrm{~L}-\mathrm{MoS}_{2}$ is twenty times lower than the needed hydrostatic compression. To the best of our knowledge, there are no high pressure studies of other monolayer TMDs under this non-hydrostatic regime and most of the existing literature is devoted to the analysis of the effects of biaxial or uniaxial in-plane strain. Wang et al. $\left.{ }^{18}\right]$ studied the strain dependence of the light emission and lattice vibration of CVD-grown monolayer WS 2 proving the tuning of different optical transition energies by applying uniaxial in-plane strain, complemented with DFT calculations. They concluded that this tunable optical property is attributed to the strain-induced direct to indirect band-gap transition. Ghosh et al. $\left.{ }^{[19}\right]$ investigated the equibiaxial strain effect on the electronic and optical properties of monolayer $\mathrm{MoSe}_{2}$ finding out that the transition indirect-to-direct bandgap semiconductor is directly related with the reduction of the monolayer thickness. It is noted that the metallization is not observed under in-plane uniaxial and equibiaxial strain regimes $\left[{ }^{18-}\right.$ 
$\left.{ }^{19}\right]$. The electronic properties have been also investigated recently for monolayer $\mathrm{WSe}_{2}$ under biaxial tensile and compressive strain using first-principles calculations $\left[{ }^{20}\right]$. The authors found out that the monolayer $\mathrm{WSe}_{2}$ retains the direct band-gap state under tension and such bandgap continuously decreases with increasing strain; and strains larger than $13 \%$ are required to reach the semimetal state. To date, the out-of-plane compression $\left[{ }^{9}\right]$ is proved to be the most effective regime to achieve the semimetal state, leading to promising future developments in strain-modulated advanced devices.

Direct out-of-plane compressive regime is used here to theoretically investigate the structural, electronic and chemical properties of mono- and bilayer TMDs. Our previous theoretical predictions of monolayer $\operatorname{MoS}_{2}\left[{ }^{9}\right]$ are, in the present work, extended to other specimens of the family $\left(\mathrm{MoSe}_{2}, \mathrm{WSe}_{2}\right.$ and $\left.\mathrm{WS}_{2}\right)$; we show a systematic analysis of the structural properties and their relationship with the modulation of the band-gap. Additionally, the chemical properties are evaluated using non-covalent interaction $(\mathrm{NCl})$ index to analyze the formation of new weak interactions induced by the direct out-of-plane compressive regime.

\section{Methodology}

First-principles calculations are performed on unstrained (pristine) and strained mono- and bilayer TMDs compounds applying direct out-of-plane compression. To this propose, the density functional theory (DFT) formalism [21,22] is used as implemented in the Vienna $A b$ initio Simulation Package (VASP) code $\left[{ }^{23}, 24\right]$. The generalized gradient approximation of Perdew, Burke and Ernzerhof (PBE) $\left[{ }^{25}\right]$ is selected to solve the Kohn-Sham equations keeping the consistency with recent studies $\left[{ }^{11,9}\right]$. Projector augmented wave (PAW) potentials $\left[{ }^{26}\right]$ are used to describe the electron-ionic core interaction and Brillouin zone integrals are approximated using Monkhorst and Pack method $\left[{ }^{27}\right]$. The energies are converged with respect the $k$-point density 10x10x1 for both mono- and bilayer systems and the plane-wave cutoff of 560 and 340 $\mathrm{eV}\left(\mathrm{MS}_{2}\right.$ and $\mathrm{MSe}_{2}$ systems, respectively) is used to ensure convergence of the total energy within $10^{-5} \mathrm{eV}$ and the geometry optimization stops when the force on each ion is less than 0.01 $\mathrm{eV} / \mathrm{A}$. The optimization of lattice parameters is calculated using the bulk unit cell of $\mathrm{MoS}_{2}, \mathrm{WS}_{2}$, 
$\mathrm{MoSe}_{2}$ and $\mathrm{WSe}_{2}$. A vacuum of $10 \AA$ to avoid the interaction between periodic slabs images along $z$-direction.

To mimic the experimental conditions of direct out-of-plane compression on monolayer compounds $\left[{ }^{9}\right]$, the $X-X$ distance decreases along the $z$-direction and $M$ atoms are allowed to relax, keeping the $a$ and $c$ lattice parameters frozen during the process. The stress, which acts on the cell, is obtained in the three directions $z$ and $x(=y)$. The in-plane/out-of-plane stress ratio is in good agreement with previous experimental evidences using moissanite anvil cells [ $\left.{ }^{28}\right]$. Different constraints are used in the case of bilayer systems where the monolayer units are moved reducing the van der Waals space between them, the external chalcogenide atoms are fixed whereas the rest of the atoms located between them are relaxed, keeping the lattice parameter constant as in the monolayer case. The out-of-plane stress values are multiplied by a correction factor considering the relationship between the unit cell top-bottom surface and the real section occupied by the atoms in the unit cell. Stress values coincided with those calculated as the energy cost over the cell volume taking the $X-X$ distance as $c$ parameter in the monolayer systems (more details over the model was recently published in $\left[{ }^{9}\right]$ ).

\section{Results and discussion}

\section{Monolayered TMDs}

The structural and electronic properties of $1 \mathrm{~L}$ TMDs in their pristine state are reported in Table 1. These results are calculated using the PBE exchange-correlation functional; it is well-known that the structural properties (i. e. lattice parameter) are overestimated whereas the electronic ones (i. e. band-gap) are underestimated with respect to the experimental data when using the generalized gradient approximation (GGA) functional as PBE. The deviations associated with the lattice parameter are below $1 \%$ (0.7 and $0.5 \%$ for $\mathrm{MoS}_{2}$ and $\mathrm{MoSe}_{2}$, respectively, and below $0.8 \%$ for $\mathrm{WS}_{2}$ and $\left.\mathrm{WSe}_{2}\right)\left[{ }^{29}\right]$. On the other hand, the band-gap is clearly underestimated, with 1.6, 6.4, 5.1 and $4.2 \%$ deviations for $\mathrm{MoS}_{2}, \mathrm{MoSe}_{2}, \mathrm{WS}_{2}$ and $\mathrm{WSe}_{2}$, respectively $\left.{ }^{9,}{ }^{30,31}\right]$. The deviation of the band-gap can be solved using hybrid functionals, which are widely used to 
investigate the electronic properties of semiconductor compounds, but the required computational time is high. For this reason, previous studies reported the modulation of the band-gap of TMDs compounds under different pressure regimes $\left.{ }^{9,11}\right]$ using the PBE functional, justifying our choice, which - as we will show next - gives reasonable results, consistent with those published. In addition, the errors generated by PBE functional do absolutely not affect the conclusions of the work.

Table 1. Structural properties of monolayer $M X_{2}$ compounds: lattice parameter, $a$, bond distances, $d(M-X), d(X-X)$, and bond angle, $\theta(X-M-X)$. The band-gap, $E_{g}$, corresponds to the direct band-gap (K-K) along the Brillouin zone. Our results are compared with experimental data available in the literature.

\begin{tabular}{|c|c|c|c|c|c|c|}
\hline Compound & & $a, \AA$ & $d(M-X), \AA$ & $d(X-X), \AA$ & $\theta(X-M-X),^{0}$ & $E_{g}, \mathrm{eV}$ \\
\hline \multirow[t]{2}{*}{$\mathrm{MoS}_{2}$} & PBE & 3.183 & 2.405 & 3.151 & 81.791 & 1.82 \\
\hline & Exp & $3.160\left[^{29}\right]$ & & & & $1.85\left[^{9}\right]$ \\
\hline \multirow[t]{2}{*}{$\mathrm{WS}_{2}$} & PBE & 3.182 & 2.416 & 3.139 & 81.013 & 1.84 \\
\hline & Exp & $3.155\left[{ }^{29}\right]$ & & & & $1.94\left[{ }^{30}\right]$ \\
\hline \multirow[t]{2}{*}{$\mathrm{MoSe}_{2}$} & PBE & 3.315 & 2.541 & 3.341 & 82.222 & 1.47 \\
\hline & Exp & $3.299\left[{ }^{29}\right]$ & & & & $1.57\left[{ }^{31}\right]$ \\
\hline \multirow[t]{2}{*}{$\mathrm{WSe}_{2}$} & PBE & 3.314 & 2.545 & 3.355 & 82.483 & 1.58 \\
\hline & $\exp$ & $3.286\left[{ }^{29}\right]$ & & & & $1.65\left[^{31}\right]$ \\
\hline
\end{tabular}

In the absence of pressure, the analysis of the band structures (black lines in Figures 1 a-d) clearly shows that the gap is governed by a direct transition with the highest and lowest energy level of the valence (VB) and the conductive (CB) bands, respectively, located in the $K$ point of the Brillouin zone. This direct band-gap energy is slightly lower than the indirect band-gap, where the VB maxima (VBM) and the $\mathrm{CB}$ minima (CBM) are located in $\Gamma$ and $K$, respectively. It must be pointed out that monolayered $\mathrm{MS}_{2}$ systems present around $0.3 \mathrm{eV}$ larger band-gaps than $\mathrm{MSe}_{2}$ monolayers, showing an inverse correlation with the $X-M-X$ bond angle, which is higher for $M \mathrm{Se}_{2}$ compounds. In fact, $X-X$ and $M-X$ distances are also lower in $\mathrm{MS}_{2}$ than in $M \mathrm{Se}_{2}$, in accordance with the bond angles (i. e. small bond angles and distances correspond to larger band gaps, and vice versa). This structural-electronic analogy may be explained using the effective ionic radius described by Shannon-Prewitt $\left.{ }^{32}\right]$. The formal $\mathrm{Se}^{2-}$ and $\mathrm{S}^{2-}$ ionic radii are 
198 and 184 pm, respectively. Thus, the monolayer with the largest chalcogenide ionic radius presents the smallest band-gap energy, due to the strong hybridization between $M$ and $X$ orbitals $\left[{ }^{33}\right]$. Other interesting issue is related with the transition metals, where MoX monolayers show lower band-gap than $W X_{2}$ ones. This fact is related with the formal transition metal ionic radius: 59 and $89 \mathrm{pm}$ for $\mathrm{Mo}^{4+}$ and $\mathrm{W}^{4+}$, respectively. And, as we will discuss next, the transition metal ionic radius plays a significant role when the monolayer compounds are subdued to direct out-of-plane compression.
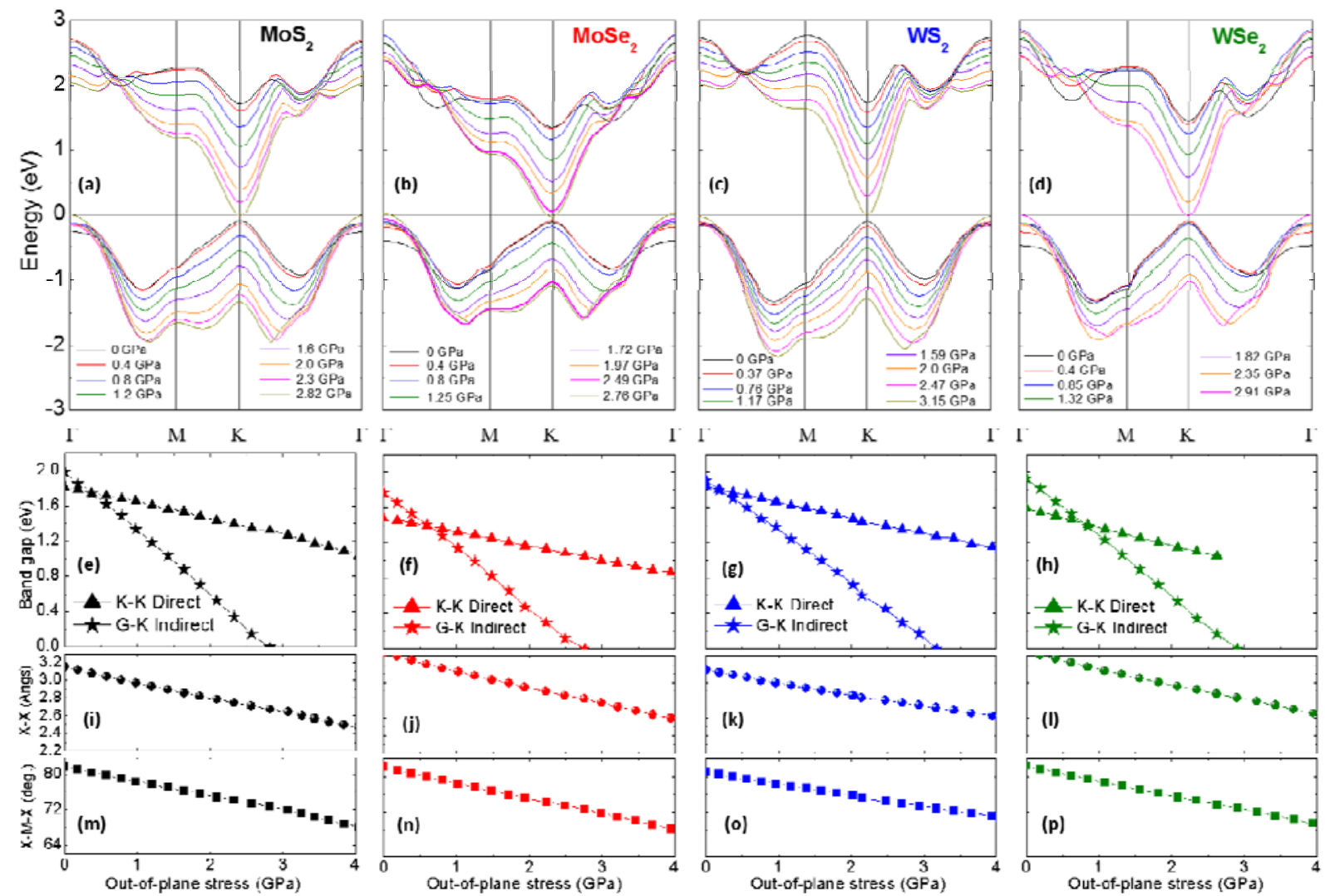

Figure 1. (a-d) Evolution of the VB and the CB under direct out-of-plane stress of $1 \mathrm{~L} M X_{2}$ monolayers (Fermi level is set to $0 \mathrm{eV})$. $(\mathrm{e}-\mathrm{h})$ Evolution of the direct $(\mathrm{K}-\mathrm{K})$ and the indirect $(\Gamma-\mathrm{K})$ band-gaps and the structural parameters: $X-X$ distance $(\mathrm{i}-\mathrm{I})$ and $X-M-X$ bond angle $(\mathrm{m}-\mathrm{p})$.

The evolution of the direct and indirect band-gaps with compression is presented in Figures 1eh. A direct-to-indirect semiconductor electronic transition takes place first, followed by the semiconductor-semimetal one (metallization process). This first electronic transition is achieved 
at $0.17,0.40,0.62$ and $0.81 \mathrm{GPa}$ for $\mathrm{WS}_{2}, \mathrm{MoS}_{2}, \mathrm{MoSe}_{2}$ and $\mathrm{WSe}_{2}$, respectively (see inset in Table 2). Sulfides transform to an indirect semiconductor state before selenides, suggesting that the compounds formed by the chalcogenide with the smaller ionic radius $\left(\mathrm{S}^{2-}<\mathrm{Se}^{2-}\right)$ requires lower compression to achieve the transition. For the particular case of monolayer $\mathrm{MoS}_{2}$ compound, we found a good agreement between the experiments and the calculations $\left.{ }^{9}\right]$ for the direct to indirect band-gap transition pressure.

The second electronic transition observed, the semimetallization, is achieved at $2.76,2.82,2.91$ and 3.15 GPa for $\mathrm{MoSe}_{2}, \mathrm{MoS}_{2}, \mathrm{WSe}_{2}$, and WS , respectively, as indicated in the inset Table in Figure 2. It is clear that monolayered $\mathrm{MoX}_{2}$ compounds undergo the semimetallization (Figure 1 e-h) before than the $W X_{2}$ ones, with the transformation governed by the ionic metal radius, $\mathrm{Mo}^{4+}<\mathrm{W}^{4+}$. Hence, there is a clear difference in the tendency for the first and the second electronic transition, as shown in Figure 2 for clarity. While the direct to indirect semiconductor transition is governed by the chalcogenide radius, in the case of the metallization the transition order is determined by the metal ionic radius, as we explain next.

In Figures $1 \mathrm{i}-\mathrm{p}$, we present the evolution of the structural parameters with the out-of-plane stress. These figures show the efficiency of such kind of compression in the reduction of the X-X perpendicular distance and the subsequent closure of the $X-M-X$ angle. It is known that the electronic structure has the main contribution from $d_{z}{ }^{2}$ (out-of-plane), $d_{x}{ }^{2}-y^{2}$ and $d_{x y}$ (in-plane) orbitals of $M$ atoms and $p$ orbitals of $X$ atoms $\left[{ }^{33}\right]$. It is noted that the VBM at $\Gamma$ and the CBM at $K$ are both dominated by $d_{z}^{2}$ and the orbital contributing to the local minimum point of the lowest conduction band along $\Gamma$ to $K$ direction exhibits strong in-plane orbitals $\left(d_{x}^{2}-y^{2}\right.$ and $\left.d_{x y}\right)$ character. Such orbital distribution is reflected in the strong correlation between the $X-M-X$ angle closure rate and the metallization stress, as presented in Figure 3; note that the metallization is reached when the CB and the VB reach the Fermi level in the $K$ and $\Gamma$ points of the Brillouin zone, respectively.

The described phenomenon would also explain the quite different reported response of monolayer $\mathrm{MoS}_{2}$ under out-of-plane and hydrostatic conditions $\left[{ }^{9,14}\right]$. The decrease of the $X-M-$ 
$X$ bond angle makes Mo $d$ orbitals interact strongly with S $p$ orbitals. This strong hybridization between the orbitals decreases the band-gap towards its closure.

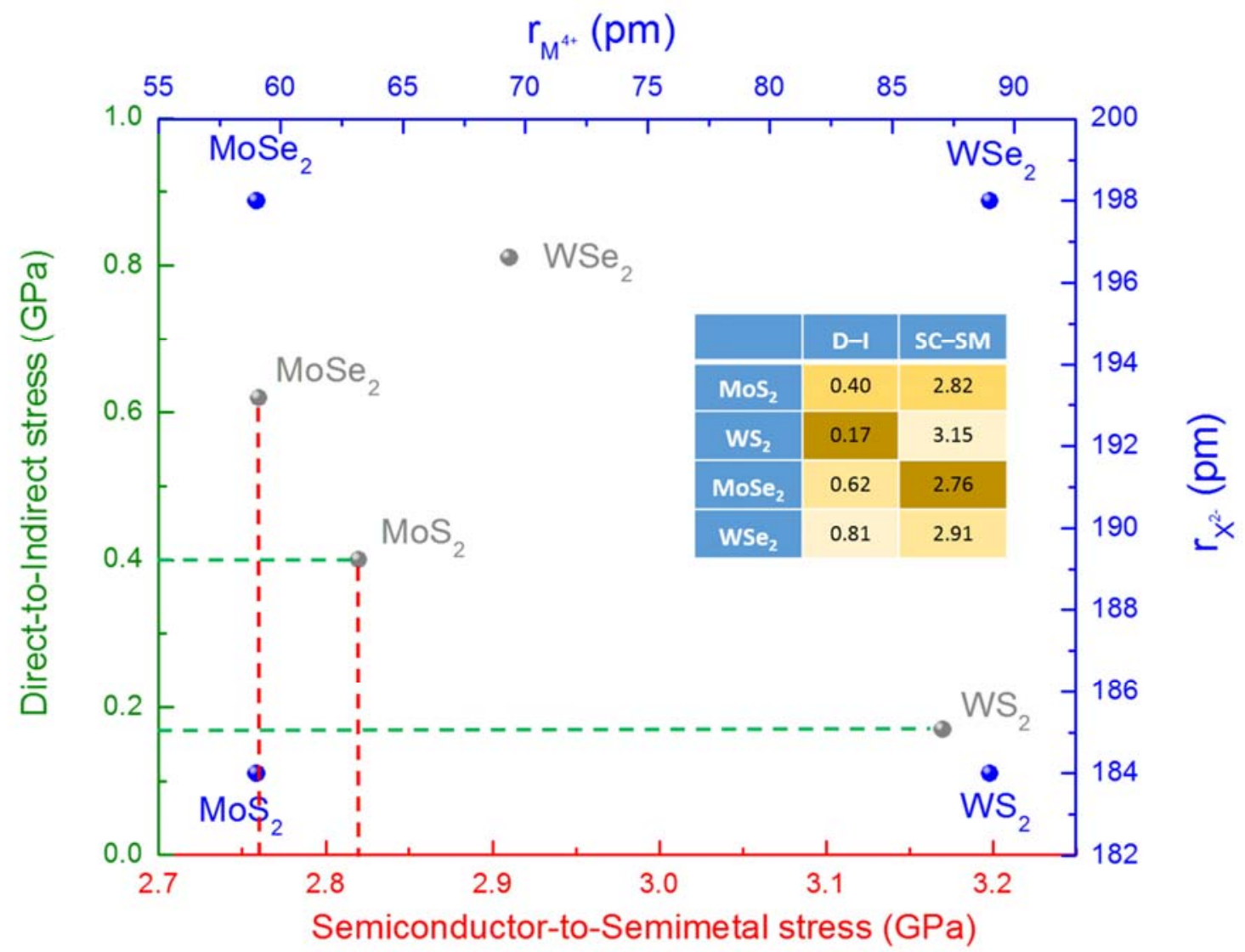

Figure 2. Blue, green and red axes correspond to atomic radii, direct-to-indirect and semiconductor-to-semimetal transition stresses, respectively. Blue spots correspond to the location of $M X_{2}$ compounds as a function of $M^{4+}$ and $X^{2-}$ radius and gray spots appear as the relation between the two electronic transitions. Red dashed lines indicate that $1 \mathrm{~L} \mathrm{MoX} X_{2}$ monolayer compounds require the smallest stress to reach the semimetal state whereas, green dashed line show that $1 \mathrm{~L} M \mathrm{~S}_{2}$ compounds undergo the transition to indirect band-gap $\mathrm{SC}$ at lower stresses than $1 \mathrm{~L}$ $\mathrm{MSe}_{2}$. The inset table shows the out-of-plane stresses at which the electronic transitions are observed. The color gradient correspond to the stress, the darkest color labels the lowest stress. 


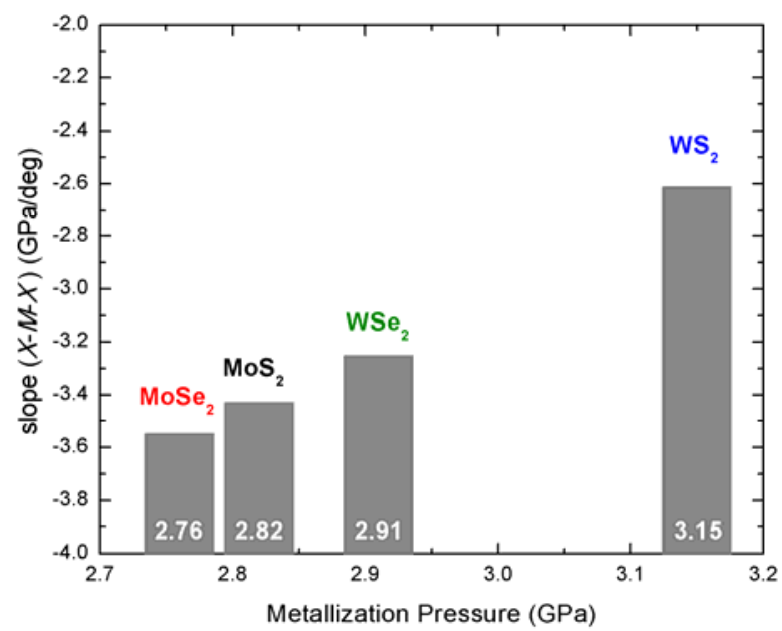

Figure 3. Relationship between the bond angle $(X-M-X)$ closure rate and the metallization stress. The white values inside the gray bars are the semimetal pressures of monolayer systems (Figure 2 inset).

\section{Bilayered TMDs}

Bilayered TMDs compounds are also investigated under the direct out-of-plane compression. The electronic properties in the pristine state of semiconductor TMDs with more than one layer are governed by the indirect band-gap. As we discussed above (monolayered TMDs), the computational band-gaps are underestimated, with deviations of $0.6,5.2,1.6$ and $3.2 \%$ for bilayered $\mathrm{MoS}_{2}, \mathrm{MoSe}_{2}, \mathrm{WS}_{2}$ and $\mathrm{WS}_{2}$, respectively (see Table 3 for the comparison between theory and experiment) $[27,34,35,36]$. In Figures $4 \mathrm{a}-\mathrm{d}$, the evolution of the VB and CB with outof-plane compression are shown for $2 \mathrm{~L}$ TMDs. In the pristine state (black lines), the VBM and the CBM are located in $\Gamma$ and $K$, respectively, for sulfur compounds $\left(M S_{2}\right)$; whereas the VBM and the CBM are located in $K$ and between $K-\Gamma$, respectively, for the selenides $\left(\mathrm{MSe}_{2}\right)$. The semimetal state is achieved at 2.07, 2.38, 2.85 and 3.55 GPa for $2 \mathrm{~L} \mathrm{MoS}_{2}, \mathrm{WS}_{2}, \mathrm{MoSe}_{2}, \mathrm{WSe}$, respectively (Figure $4 \mathrm{e}-\mathrm{h}$ and Table 3 ). An intriguing behavior is observed when comparing the metallization stress of $1 \mathrm{~L}$ and $2 \mathrm{~L}$ systems: within $1 \mathrm{~L}$ TMDs the metal state is achieved at lower stress by the $\mathrm{MoX}_{2}$ compounds, whereas in the case of $2 \mathrm{~L}$ specimens sulfides metalize before than selenides. Moreover, we observe that the bilayered sulfides metallize at lower pressure 
than their monolayered counterpart; however, this behavior is opposite for selenide compounds.

Table 3. Theoretical and experimental indirect band-gap of 2L-TMDs (in eV) along with the values of metallization stress. Our results are compared with experimental data available in the literature.

\begin{tabular}{|c|c|c|c|c|c|}
\hline \multicolumn{2}{|c|}{ Bilayer } & MoS $_{\mathbf{2}}$ & WS $_{\mathbf{2}}$ & MoSe $_{\mathbf{2}}$ & WSe $_{\mathbf{2}}$ \\
\hline $\begin{array}{c}\text { Indirect band-gap } \\
(\mathrm{eV})\end{array}$ & Present work & 1.59 & 1.79 & 1.44 & 1.53 \\
\cline { 2 - 6 } & experimental & $1.60\left[{ }^{34}\right]$ & $1.82\left[{ }^{35}\right]$ & $1.52\left[{ }^{27}\right]$ & $1.58\left[{ }^{36}\right]$ \\
\hline \multicolumn{2}{|c|}{ Semiconductor-to-Semimetal (GPa) } & 2.07 & 2.38 & 2.85 & 3.55 \\
\hline
\end{tabular}
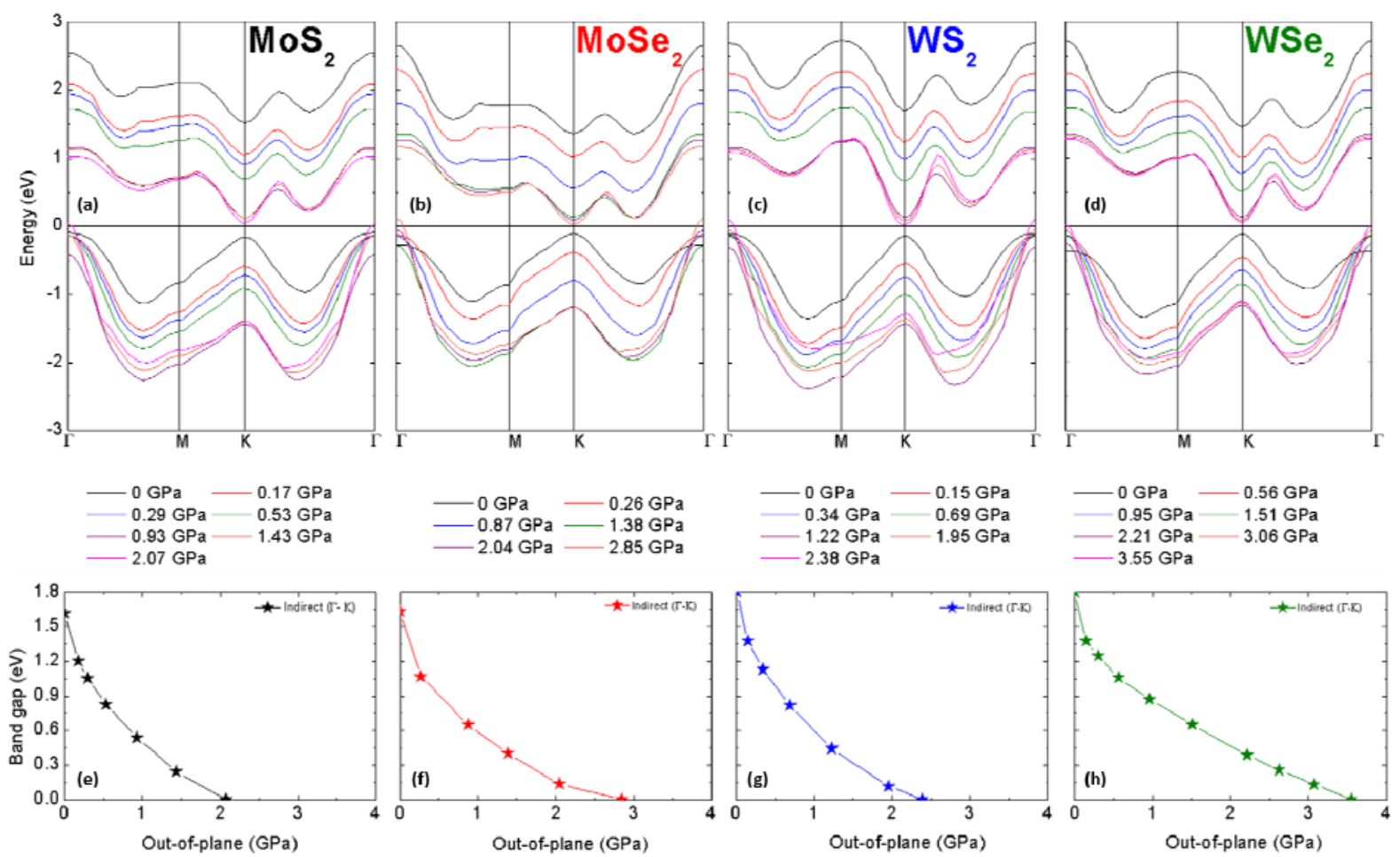

Figure 4. (a-d) Evolution of the VB and the CB bands under direct out-of-plane stress of 2LTMDs (Fermi level is set to $E=0 \mathrm{eV}$ ) at PBE level. (e-h) Evolution of the band gap (indirect) towards semimetal state under compression.

Such intriguing behavior can be explained in view of the evolution of the structural parameters with stress. In the case of $2 \mathrm{~L} M X_{2}$ we are not able to find a structural indicator of the metallization as clear as in the case of the analogous monolayers; the evolution of the structural parameters (Figure 5) is analogous for all systems; and simply, those bilayered systems with smaller bond angles and distances in the pristine state metallize at lower 
pressures. In this regard, the parameters of the bilayered sulfide compounds are systematically below the selenide ones. Interestingly, among all structural parameters, the interlayer distance, labeled as $d(X-X)_{\text {inter, }}$ shows the most significant changes with compression, indicating that the van der Waals space between the layers is the feature most sensitive to the effect of out-ofplane stress. Looking at Figure $5 \mathrm{e}$, there is an important reduction of this parameter at the initials stages of compression (below $0.5 \mathrm{GPa}$ ), favoring the interaction between the orbitals [11]. This reduction is higher for sulfide systems, promoting an earlier metallization than in the selenide analogues. Hence, the changes in the electronic properties are attributed to the hybridization between out-of-plane orbitals of different layers $\left[{ }^{37}\right]$ due to the effect of pressure. Following this idea, the differences of hybridization are directly related with the atomic chalcogenide radius: $\mathrm{S}^{2-}$ radius $(184 \mathrm{pm})$ and $\mathrm{Se}^{2-}(198 \mathrm{pm})$. The bilayered compounds formed by $S$ atoms promote a stronger hybridization of the orbitals between the layers leading to a semimetallization at lower compression, below 2.4 GPa. Under the application of direct out-ofplane pressure, the interlayer distance between the atomic planes decreases and the intra- as well as interlayer interactions alter the electronic properties of TMDs.
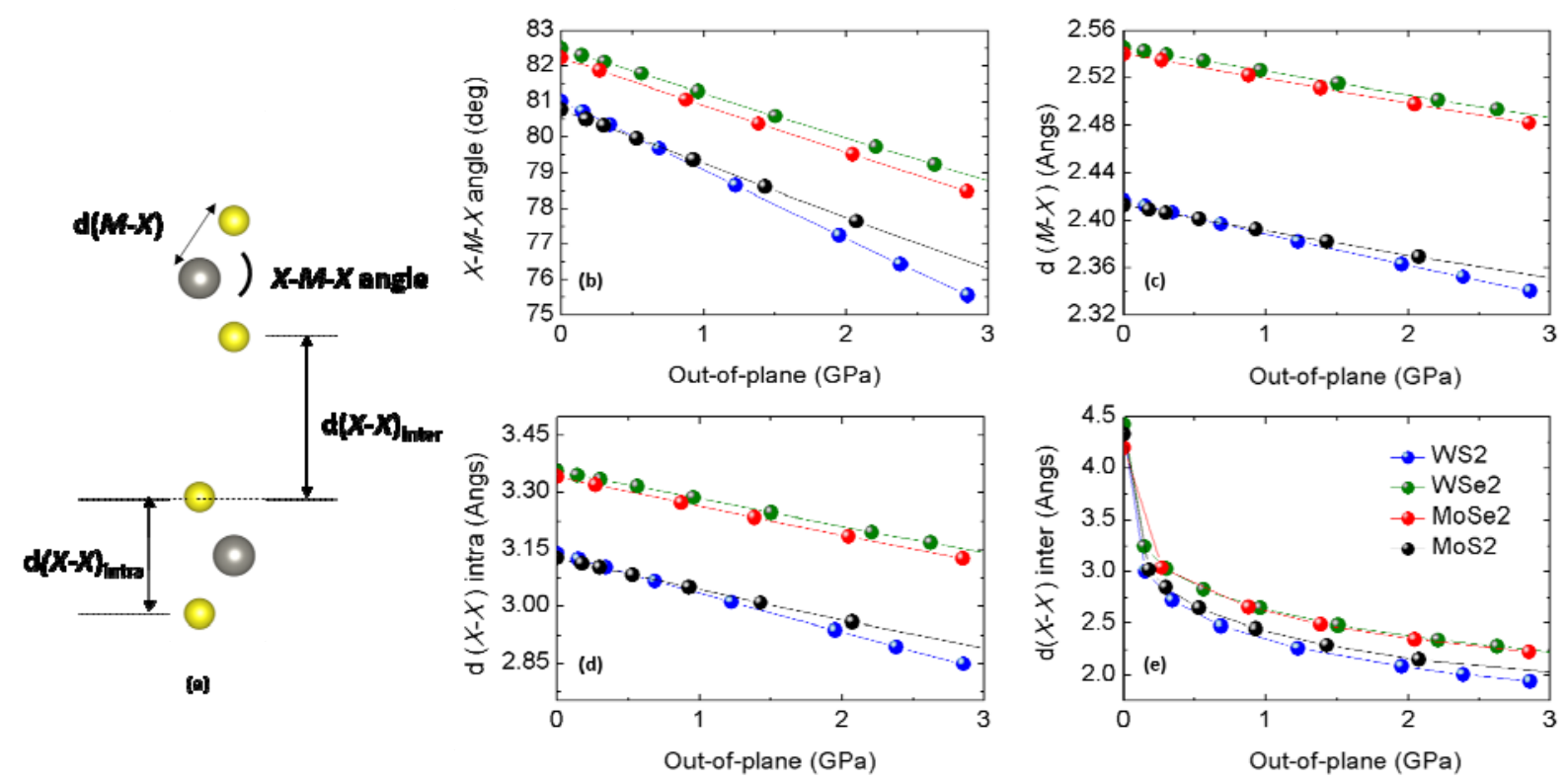

Figure 5. (a) Structural parameters of $M X_{2}$ bilayer compounds evaluated under out-of-plane compression: (b) $X-M$ $X$ bond angle, (c) $M-X$ distance, and (d) intra and (e) inter $X-X$ distances. 


\section{Non-covalent domains, chemical properties}

The semimetal state of mono- and bilayered $M X_{2}$ compounds is explained using the so-called non-covalent interactions $(\mathrm{NCl})$ index[ $\left.{ }^{38-39}\right]$, which is based on the reduced electron density gradient (RDG, s). The $\mathrm{NCl}$ approach has shown great success for visualizing weak interactions and enabling the characterization of both stabilizing (hydrogen bonds and $\mathrm{vdW}$ ) and destabilizing (steric clashes) interactions in different materials $\left[{ }^{40,41}\right]$. These interactions can be highlighted within the molecular frame by means of $s$ isosurfaces (Figure 6). In order to visually identify the interaction type, a color code is used [ $\left.{ }^{38}\right]$ : blue for attractive, green for vdW and red for clashes. The $\mathrm{NCl}$ analysis is carried out with the CRITIC2 code $\left[{ }^{42}\right]$ and the $\mathrm{NCl}$ domains are then visualized using VMD 1.9.1 $\left[{ }^{43}\right]$. Recently, $\mathrm{NCl}$ index has been used to explain the metallization of semiconductor compounds under different strain regimes $\left[{ }^{41}\right]$.

This analysis has been performed for all mono- and bilayer compounds, the information obtained is qualitatively analogous to the case of $\mathrm{MoS}_{2}$ (Figure 6). In monolayer systems (Figure 6a) the $\mathrm{NCl}$ domains increase in the $\mathrm{S} \cdots \mathrm{S}$ space (blue iso-surface) by the effect of strain promoting the attraction between the sulfur atoms. On the other hand, the $\mathrm{NCl}$ domains appear in the interlayer space of the bilayer compounds (Figure 6b). These $\mathrm{NCl}$ domains are not present in the pristine state and they are related with the changes of the electronic properties discussed above.

(a)

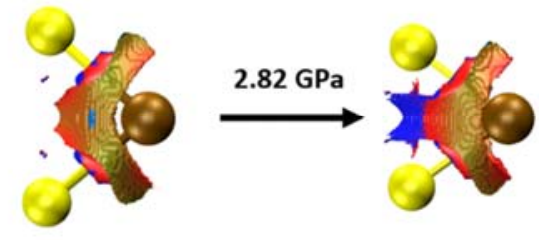

2L

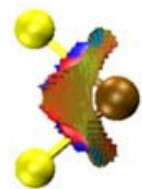

(b)

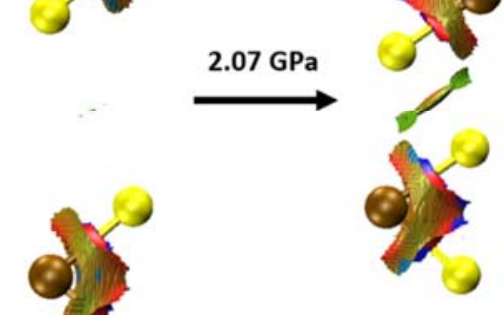

Figure 6. Domains of the reduced density gradient (RDG, $s=0.4$ a.u.) for $1 \mathrm{~L}-\mathrm{MoS}_{2}$ (a) and 2L-MoS (b). Brown and yellow spheres correspond to $\mathrm{Mo}$ and $\mathrm{S}$ atoms, respectively. 


\section{Conclusions}

The modulation of band-gap is systematically studied by means of first-principles calculations for the TMDs family: $\mathrm{MoS}_{2}, \mathrm{MoSe}_{2}, \mathrm{WS}_{2}$ and $\mathrm{WSe}_{2}$ (mono- and bilayer systems) under direct out-of-plane compression. This particular regime, in contrast to hydrostatic compression, reduces significantly the stress range at which the semimetal state is achieved. Two electronic transitions are reported for the monolayer systems. (i) The direct to indirect band-gap (below 1 $\mathrm{GPa}$ ); this transition is reached in sulfides sooner than in selenides indicating a direct correlation with the chalcogenide atomic radius $\left(\mathrm{S}^{2-}<\mathrm{Se}^{2-}\right)$. (ii) The semiconductor to semimetal transition is achieved at $\sim 3 \mathrm{GPa}$; in this case, $\mathrm{MoX}_{2}$ systems show lower transition stress threshold than $W X_{2}$; indicating that the hybridization of the out-of-plane orbitals is more effective in the TMDs monolayers formed by the transition metal with smaller ionic radius $\left(\mathrm{Mo}^{4+}<\mathrm{W}^{4+}\right)$. On the other hand, the van der Waals space between the layers is the key parameter for understanding the transition to the semimetal state in bilayer systems. Here, the hybridization of the orbitals between the layers promotes the transition to the semimetal state and it is governed by the chalcogenide ion radius. Finally, the $\mathrm{NCl}$ index analysis confirms that the appearance of new non-covalent domains is related with the electronic changes induced by direct out-of-plane compression, reporting the consistency between the structural, electronic and chemical properties. Our predictions can be of a great utility for the experimentalist since the transition stresses reported here are easily reached using DAC devices. Our results also open plausible new ways for the synthesis of semiconductor TMDs leading to an easy modulation of the band-gap under stress.

\section{Acknowledgements}

Computational resources from Universidad de Oviedo (MALTA Supercomputing Center) provided the required computational. A. M.-G acknowledges the computer resources, technical expertise and assistance provided by the Barcelona Supercomputing Center - Centro Nacional de Supercomputación (QCM-2016-1-0019). E.d.C and O.F. acknowledge the support of the Czech Science Foundation project 14-15357S. 


\section{Uncategorized References}

1. Wang, Q. H.; Kalantar-Zadeh, K.; Kis, A.; Coleman, J. N.; Strano, M. S., Electronics and optoelectronics of two-dimensional transition metal dichalcogenides. Nat Nanotechnol 2012, 7 (11), 699-712.

2. Zhang, Y. J.; Ye, J. T.; Matsuhashi, Y.; Iwasa, Y., Ambipolar MoS2 Thin Flake Transistors. Nano Lett 2012, 12 (3), 1136-1140.

3. Jain, J. R.; Hryciw, A.; Baer, T. M.; Miller, D. A. B.; Brongersma, M. L.; Howe, R. T., A micromachining-based technology for enhancing germanium light emission via tensile strain. Nat Photonics 2012, 6 (6), 398-405.

4. Castellanos-Gomez, A.; Roldan, R.; Cappelluti, E.; Buscema, M.; Guinea, F.; van der Zant, H. S. J.; Steele, G. A., Local Strain Engineering in Atomically Thin MoS2. Nano Lett 2013, 13 (11), 5361-5366.

5. Conley, H. J.; Wang, B.; Ziegler, J. I.; Haglund, R. F.; Pantelides, S. T.; Bolotin, K. I., Bandgap Engineering of Strained Monolayer and Bilayer MoS2. Nano Lett 2013, 13 (8), 3626-3630.

6. Dou, X. M.; Ding, K.; Jiang, D. S.; Sun, B. Q., Tuning and Identification of Interband Transitions in Monolayer and Bilayer Molybdenum Disulfide Using Hydrostatic Pressure. Acs Nano 2014, 8 (7), 74587464.

7. Espejo, C.; Rangel, T.; Romero, A. H.; Gonze, X.; Rignanese, G. M., Band structure tunability in MoS2 under interlayer compression: A DFT and GW study. Phys Rev B 2013, 87 (24).

8. Guo, H. Y.; Lu, N.; Wang, L.; Wu, X. J.; Zeng, X. C., Tuning Electronic and Magnetic Properties of Early Transition-Metal Dichalcogenides via Tensile Strain. Journal of Physical Chemistry C 2014, 118 (13), 7242-7249.

9. Peña-Alvarez, M.; del Corro, E.; Morales-Garcia, A.; Kavan, L.; Kalbac, M.; Frank, O., Single Layer Molybdenum Disulfide under Direct Out-of-Plane Compression: Low-Stress Band-Gap Engineering. Nano Lett 2015, 15 (5), 3139-3146.

10. Amin, B.; Kaloni, T. P.; Schwingenschlogl, U., Strain engineering of WS2, WSe2, and WTe2. RsC Advances 2014, 4 (65), 34561-34565.

11. Nayak, A. P.; Bhattacharyya, S.; Zhu, J.; Liu, J.; Wu, X.; Pandey, T.; Jin, C. Q.; Singh, A. K.; Akinwande, D.; Lin, J. F., Pressure-induced semiconducting to metallic transition in multilayered molybdenum disulphide. Nat Commun 2014, 5.

12. Kumar, A.; Ahluwalia, P. K., Semiconductor to metal transition in bilayer transition metals dichalcogenides MX2 (M = Mo, W; X = S, Se, Te). Model Simul Mater Sc 2013, 21 (6).

13. Su, X. Y.; Zhang, R. Z.; Guo, C. F.; Zheng, J. M.; Ren, Z. Y., Band engineering of dichalcogenide MX2 nanosheets ( $M=$ Mo, $W$ and $X=S, S e)$ by out-of-plane pressure. Phys Lett $A$ 2014, 378 (9), 745-749.

14. Nayak, A. P.; Pandey, T.; Voiry, D.; Liu, J.; Moran, S. T.; Sharma, A.; Tan, C.; Chen, C. H.; Li, L. J.; Chhowalla, M.; Lin, J. F.; Singh, A. K.; Akinwande, D., Pressure-Dependent Optical and Vibrational Properties of Mono layer Molybdenum Disulfide. Nano Lett 2015, 15 (1), 346-353.

15. Zhao, Z.; Zhang, H. J.; Yuan, H. T.; Wang, S. B.; Lin, Y.; Zeng, Q. S.; Xu, G.; Liu, Z. X.; Solanki, G. K.; Patel, K. D.; Cui, Y.; Hwang, H. Y.; Mao, W. L., Pressure induced metallization with absence of structural transition in layered molybdenum diselenide. Nat Commun 2015, 6.

16. Nayak, A. P.; Yuan, Z.; Cao, B. X.; Liu, J.; Wu, J. J.; Moran, S. T.; Li, T. S.; Akinwande, D.; Jin, C. Q.; Lin, J. F., Pressure-Modulated Conductivity, Carrier Density, and Mobility of Multi layered Tungsten Disulfide. Acs Nano 2015, 9 (9), 9117-9123.

17. Liu, B.; Han, Y. H.; Gao, C. X.; Ma, Y. Z.; Peng, G.; Wu, B. J.; Liu, C. L.; Wang, Y.; Hu, T. J.; Cui, X. Y.; Ren, W. B.; Li, Y.; Su, N. N.; Liu, H. W.; Zou, G. T., Pressure Induced Semiconductor-Semimetal Transition in WSe2. Journal of Physical Chemistry C 2010, 114 (33), 14251-14254. 
18. Wang, Y. L.; Cong, C. X.; Yang, W. H.; Shang, J. Z.; Peimyoo, N.; Chen, Y.; Kang, J. Y.; Wang, J. P.; Huang, W.; Yu, T., Strain-induced direct-indirect bandgap transition and phonon modulation in monolayer WS2. Nano Res 2015, 8 (8), 2562-2572.

19. Ghosh, C. K.; Sarkar, D.; Mitra, M. K.; Chattopadhyay, K. K., Equibiaxial strain: tunable electronic structure and optical properties of bulk and monolayer MoSe2. J Phys D Appl Phys 2013, 46 (39).

20. Yang, C. Z., X.; Wei S., Manipulation of electronic structure in WSe2 monolayer by strain. Solid State Communications 2016.

21. Hohenberg, P.; Kohn, W., Inhomogeneous Electron Gas. Phys Rev B 1964, 136 (3b), B864--.

22. Kohn, W.; Sham, L. J., Self-Consistent Equations Including Exchange and Correlation Effects. Phys Rev 1965, 140 (4a), 1133-\&.

23. Kresse, G.; Furthmuller, J., Efficiency of ab-initio total energy calculations for metals and semiconductors using a plane-wave basis set. Comp Mater Sci 1996, 6 (1), 15-50.

24. Kresse, G.; Furthmuller, J., Efficient iterative schemes for ab initio total-energy calculations using a plane-wave basis set. Phys Rev B 1996, 54 (16), 11169-11186.

25. Perdew, J. P.; Burke, K.; Ernzerhof, M., Generalized gradient approximation made simple. Physical Review Letters 1996, 77 (18), 3865-3868.

26. Blochl, P. E., Projector Augmented-Wave Method. Phys Rev B 1994, 50 (24), 17953-17979.

27. Ji, J.; Zhang, A.; Xia, T.; Gao, P.; Jie, Y.; Zhang, Q.; Zhang, Q., Strain-modulated excitonic gaps in mono- and bi-layer MoSe2. Chinese Physical B 2016, 25 (7), 077802(1)-077802(4).

28. del Corro, E.; Izquierdo, J. G.; Gonzalez, J.; Taravillo, M.; Baonza, V. G., 3D Raman mapping of uniaxially loaded 6H-SiC crystals. J Raman Spectrosc 2013, 44 (5), 758-762.

29. Yun, W. S.; Han, S. W.; Hong, S. C.; Kim, I. G.; Lee, J. D., Thickness and strain effects on electronic structures of transition metal dichalcogenides: $2 \mathrm{H}-\mathrm{M} \mathrm{X}-2$ semiconductors $(\mathrm{M}=\mathrm{Mo}, \mathrm{W} ; \mathrm{X}=\mathrm{S}, \mathrm{Se}, \mathrm{Te})$. Phys Rev B 2012, 85 (3).

30. Wang, X. H.; Ning, J. Q.; Zheng, C. C.; Zhu, B. R.; Xie, L.; Wu, H. S.; Xu, S. J., Photoluminescence and Raman mapping characterization of WS2 monolayers prepared using top-down and bottom-up methods. J Mater Chem C 2015, 3 (11), 2589-2592.

31. Tonndorf, P.; Schmidt, R.; Bottger, P.; Zhang, X.; Borner, J.; Liebig, A.; Albrecht, M.; Kloc, C.; Gordan, O.; Zahn, D. R. T.; de Vasconcellos, S. M.; Bratschitsch, R., Photoluminescence emission and Raman response of monolayer MoS2, MoSe2, and WSe2. Opt Express 2013, 21 (4), 4908-4916.

32. Shannon, R. D., Revised Effective Ionic-Radii and Systematic Studies of Interatomic Distances in Halides and Chalcogenides. Acta Crystallogr A 1976, 32 (Sep1), 751-767.

33. Chang, C. H.; Fan, X. F.; Lin, S. H.; Kuo, J. L., Orbital analysis of electronic structure and phonon dispersion in MoS2, MoSe2, WS2, and WSe2 monolayers under strain. Phys Rev B 2013, 88 (19).

34. Chu, T.; llatikhameneh, H.; Klimeck, G.; Rahman, R.; Chen, Z. H., Electrically Tunable Bandgaps in Bilayer MoS2. Nano Lett 2015, 15 (12), 8000-8007.

35. Jo, S.; Ubrig, N.; Berger, H.; Kuzmenko, A. B.; Morpurgo, A. F., Mono- and Bilayer WS2 LightEmitting Transistors. Nano Lett 2014, 14 (4), 2019-2025.

36. Desai, S. B.; Seol, G.; Kang, J. S.; Fang, H.; Battaglia, C.; Kapadia, R.; Ager, J. W.; Guo, J.; Javey, A., Strain-Induced Indirect to Direct Bandgap Transition in Multi layer WSe2. Nano Lett 2014, 14 (8), 45924597.

37. Fang, S. A.; Defo, R. K.; Shirodkar, S. N.; Lieu, S.; Tritsaris, G. A.; Kaxiras, E., Ab initio tight-binding Hamiltonian for transition metal dichalcogenides. Phys Rev B 2015, 92 (20).

38. Johnson, E. R.; Keinan, S.; Mori-Sanchez, P.; Contreras-Garcia, J.; Cohen, A. J.; Yang, W. T., Revealing Noncovalent Interactions. J Am Chem Soc 2010, 132 (18), 6498-6506.

39. Contreras-Garcia, J.; Johnson, E. R.; Keinan, S.; Chaudret, R.; Piquemal, J. P.; Beratan, D. N.; Yang, W. T., NCIPLOT: A Program for Plotting Noncovalent Interaction Regions. J Chem Theory Comput 2011, 7 (3), 625-632. 
40. Christian, M. S.; Whittleton, S. R.; Otero-de-la-Roza, A.; Johnson, E. R., Chemical bonding and surface interactions in Bi2Se3 and Bi4Se3. Comput Theor Chem 2015, 1053, 238-244.

41. Guedda, H. Z.; Ouahrani, T.; Morales-Garcia, A.; Franco, R.; Salvado, M. A.; Pertierra, P.; Recio, J. M., Computer simulations of $3 \mathrm{C}-\mathrm{SiC}$ under hydrostatic and non-hydrostatic stresses. Physical Chemistry Chemical Physics 2016, 18 (11), 8132-8139.

42. Otero-de-la-Roza, A.; Johnson, E. R.; Contreras-Garcia, J., Revealing non-covalent interactions in solids: NCl plots revisited. Phys Chem Chem Phys 2012, 14 (35), 12165-12172.

43. Humphrey, W.; Dalke, A.; Schulten, K., VMD: Visual molecular dynamics. J Mol Graph Model 1996, 14 (1), 33-38. 